\title{
Precoated brackets offer no clinical advantage over non-precoated brackets
}

\author{
Is there any difference between precoated and non-precoated brackets with \\ respect to bond failure rate and time taken in the dental practice to place \\ brackets?
}

\section{Wong M, Power S. A prospective randomised clinical trial to compare pre-coated and non-pre-coated brackets. J Orthod 2003; 30:155-158}

Design This was a randomised controlled trial (RCT) in the orthodontic department of a UK National Health Service hospital.

Intervention Patients' teeth were bonded using a split-mouth technique, ie, randomly allocating the precoated brackets to upper left and lower right quadrants, and non-precoated brackets to the other quadrants.

Outcome measure The clinical time required for bond up of upper and lower arches of both systems and the bond failure rate for the first 6 months were measured.

Results A total of 33 patients were entered into the trial and 746 brackets (372 precoated and 374 non-precoated) were placed. There were no significant differences in either the clinical time required to place precoated brackets or bond failure rate compared with nonprecoated brackets.

Conclusions Neither the precoated nor the non-precoated brackets evaluated were clinically superior to the others in terms of bond failure rate in the first 6 months of fixed appliance treatment. The use of precoated brackets did not result in a significant reduction in clinical bonding time.

\section{Commentary}

The purpose of this RCT was straightforward: to find out if there were clinical advantages of precoated brackets over non-precoated ones. Efficient orthodontic treatment with a fixed appliance is dependent on bracket/adhesive systems having adequate bond strength. In the past, many clinical trials have been performed with the aim of assessing different adhesives, methods of curing and tooth preparation procedures. ${ }^{1}$ Bonding strength, however, is also dependent on the surface characteristics of the brackets. Some of the bonding failure happens at the adhesive-bracket interface, which means the adhesive is strong enough to hold on the tooth surface (chemical and physical bond) but not on the bracket surface (only physical bond). ${ }^{2}$ Thus, it is worthwhile to have the brackets precoated with adhesives which theoretically can improve the bond strength at the adhesive-bracket interface.

This RCT used a split-mouth design on 746 brackets in 33 patients. The results showed that the precoated brackets did not in fact demonstrate any clinical advantages in terms of 'chair time' and bond failure in the first 6 months of treatment. It would be interesting, however, to follow up the patients for a longer period. This should be possible since the brackets will not be debonded after the first 6 months of study.

\section{Practice point}

- No clinical advantage seen with precoated brackets in the first 6 months of treatment but longer term results are required.

\section{Yijin Ren \\ Orthodontic Department, University Hospital Gröningen, Gröningen, The Netherlands}

1. Harrison JE. Clinical trials in orthodontics I: demographic details of clinical trials published in three orthodontic journals between 1989 and 1998. J Orthod 2003; 30:25-30.

2. Zachrisson BU. Bonding in orthodontics Chapter in Orthodontics: Current Principles and Techniques. 2nd edn. Edited by Graber TM and Vanarsdall RL. St Louis: Mosby Year Book; 1994; 542-626.

Evidence-Based Dentistry (2004) 5, 104.

doi:10.1038/sj.ebd.6400287 